\title{
Accuracy Evaluation for Region Centroid-Based Registration of Fluorescent CLSM Imagery
}

\author{
Sang-Chul Lee, ${ }^{1}$ Peter Bajcsy, ${ }^{1}$ Amy Lin, ${ }^{2}$ and Robert Folberg ${ }^{2}$ \\ ${ }^{1}$ The National Center for Supercomputing Applications, University of Illinois at Urbana-Champaign, Urbana, IL 61801, USA \\ ${ }^{2}$ Department of Pathology, The University of Illinois Cancer Center, University of Illinois at Chicago, Chicago, IL 60607, USA
}

Received 1 March 2005; Revised 30 September 2005; Accepted 16 November 2005

We present an accuracy evaluation of a semiautomatic registration technique for $3 \mathrm{D}$ volume reconstruction from fluorescent confocal laser scanning microscope (CLSM) imagery. The presented semiautomatic method is designed based on our observations that (a) an accurate point selection is much harder than an accurate region (segment) selection for a human, (b) a centroid selection of any region is less accurate by a human than by a computer, and (c) registration based on structural shape of a region rather than based on intensity-defined point is more robust to noise and to morphological deformation of features across stacks. We applied the method to image mosaicking and image alignment registration steps and evaluated its performance with 20 human subjects on CLSM images with stained blood vessels. Our experimental evaluation showed significant benefits of automation for 3D volume reconstruction in terms of achieved accuracy, consistency of results, and performance time. In addition, the results indicate that the differences between registration accuracy obtained by experts and by novices disappear with the proposed semiautomatic registration technique while the absolute registration accuracy increases.

Copyright @ 2006 Hindawi Publishing Corporation. All rights reserved.

\section{INTRODUCTION}

The problem of $3 \mathrm{D}$ volume reconstruction can be found in multiple application domains, such as medicine, mineralogy, or surface material science. In almost all applications, the overarching goal is to automate a $3 \mathrm{D}$ volume reconstruction process while achieving at least the accuracy of a human operator. The benefits of automation include not only the cost of human operators but also the improved consistency of reconstruction and the eliminated training time of operators. Thus, in this paper, we study the performance of fully automatic, semiautomatic, and manual 3D volume reconstruction methods in a medical domain [1]. Specifically, we conduct experiments with fluorescent confocal laser scanning microscope imagery used for mapping the distribution of extracellular matrix proteins in serial histological sections of uveal melanoma $[2,3]$.

In general, a feature-based 3D volume reconstruction without a priori information requires performing the following steps. First, select a reference coordinate system or a reference image. Second, determine location of salient features in multiple data sets. This step is also denoted as finding spatial correspondences. Third, select a registration transformation model that will compensate for geometric distortions. Fourth, evaluate registration accuracy with a selected metric.
Regardless of the automation category (manual or semiautomatic), these selections and evaluations are needed to perform 3D volume reconstruction. The challenges lie not only in making appropriate selections in the aforementioned steps but also in defining optimality criteria for any made selection. In many cases, it is very hard to assess the registration accuracy objectively due to a lack of a priori information about data sets. While the selection challenges are one part of each registration technique, the accuracy assessment challenge is addressed in the experimental evaluation.

There exist many techniques for 3D volume reconstruction and many commercial tools from multiple vendors that could be used for image registration [4-9]. An overview of 3D registration tools for MRI, CT, confocal, and serialsection data for medical/life-sciences imaging is provided at the Stanford or at the NIH web sites (http://biocomp.stanford.edu/3dreconstruction/software/ and http://www.mwrn. $\mathrm{com} /$ guide/image/analysis.htm). One could list some of the few software tools that have been developed specifically for CLSM, for example, 3D-Doctor, Science GL, MicroVoxel, 3DVIEWNIX, or Analyze. Most of these tools use manual registration methods, and users have to make manual selections, as described in the previous paragraph, before any particular software reports registration error associated with registered images. Some software packages include 
semiautomatic or fully automatic 3D volume re-con-struction for specific imaging modalities under the assumption that visually salient markers have been inserted artificially in imaged specimens. For instance, 3D-Doctor provides a maximum likelihood algorithm for aligning slices under such assumption.

This paper presents evaluations that are of interest to researchers who have done similar work but never had the time to quantify the pros and cons of (1) automation level, (2) expertise level, and (3) transformation model complexity variables for mosaicking and image alignment registration problems. In addition, while the registration techniques used in our work are well known, they have been applied in the past to other imaging modalities, for example, MRI, CT, PET, than the fluorescent CLSM imagery. The specific challenges of fluorescent CLSM imaging, 3D volume reconstruction without fiduciary markers, and designing an evaluation methodology have to be understood when the standard registration algorithms are applied. We provide such results for the benefit of the researchers that work with or consider using CLSM imaging modality.

Our proposed work aims at estimating upper error bounds for automatic, semiautomatic, and manual 3D volume reconstruction techniques. To achieve our aim, we have developed three mosaicking methods (registration of $x-y$ image tiles in a single frame of a physical section) and two alignment algorithms (registration of $z$-slides from multiple physical sections). Next, we designed an experimental evaluation methodology that addresses the issues of (a) defining optimality criteria for assessing registration accuracy and (b) obtaining the ground truth (or reference) images, as encountered in real medical registration scenarios. After conducting experiments with human subjects consisting of experts and novices, we drew conclusions about the $3 \mathrm{D}$ reconstruction methods and thoroughly analyzed the driving factors behind our results.

This paper is organized in the following way. Section 2 introduces the 3D volume reconstruction problem on CLSM images. Section 3 describes all image mosaicking and alignment registration methods developed for the accuracy assessment study. Section 4 presents our evaluation methodology for multiple registration methods. Finally, all experimental results are presented and analyzed in Section 5, and our work is summarized in Section 6.

\section{PROBLEM STATEMENTS}

We define the $3 \mathrm{D}$ reconstruction problem as a registration problem [10]. The goal of $3 \mathrm{D}$ reconstruction is to form a high-resolution 3D volume with large spatial coverage from a set of spatial tiles (small spatial coverage and high-resolution $2 \mathrm{D}$ images or $3 \mathrm{D}$ cross-section volumes). $3 \mathrm{D}$ volumetric data are acquired from multiple cross-sections of a tissue specimen by (a) placing each cross-section under a laser scanning confocal microscope, (b) changing the focal length to obtain an image stack per cross-section, and (c) moving the specimen spatially for specimen location. The set of spatial tiles is acquired by CLSM and consists of images that came from one

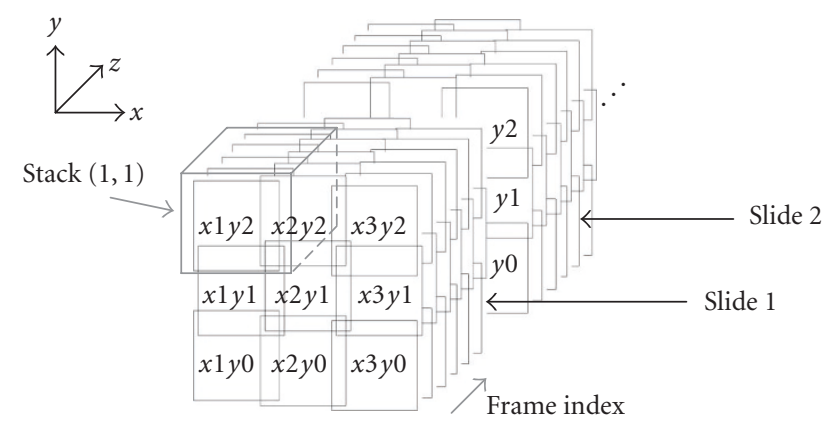

FIGURE 1: An overview of 3D volume reconstruction from fluorescent laser scanning confocal microscope images.

or multiple cross-sections of a 3D volume. Our objectives are to (1) mosaic (stitch together) spatial tiles that came from the same cross-section, (2) align slides (physical sections) from multiple cross-sections, and (3) evaluate the accuracy of $3 \mathrm{D}$ volume reconstruction using multiple techniques. An overview of the $3 \mathrm{D}$ volume reconstruction problem is illustrated in Figure 1. Our assumption is that there is no prior information about (a) tile locations and their spatial overlap, (b) cross-section feature, and (c) evaluation methodology and metrics.

It is apparent that using artificially inserted fiduciary markers allows automating 3D volume reconstruction while keeping the registration error low. However, there still exist medical experiments with CLSM, where fiduciary markers cannot be inserted into a specimen. For example, the placement of fiduciary markers in paraffin-embedded tissue is problematic. The introduction of markers internally may distort tissue and areas of interest. On the other hand, markers placed outside the tissue may migrate during sectioning or expansion of the paraffin. The composition of the marker also poses challenges. Rigid material, such as suture, may fragment or distort the tissue when sections are cut. In addition to attempting to locate fiduciary markers into tissues using the aforementioned techniques, it is also attempted to insert small cylindrical segments of "donor tissue" from paraffin-embedded tissues according to the techniques used to construct tissue microarrays [11]. It is discovered that the round outlines of donor tissue cores were inconsistent between tissue sections, making it impossible to use these donor samples as reliable internal fiduciary markers.

Although we are addressing the $3 \mathrm{D}$ volume reconstruction problem without artificially inserted fiduciary markers into paraffin-embedded tissue, we still need to identify an internal specimen structure for registration that would be visually salient. For this purpose, tonsil tissue was selected because it contained structures of interest, for example, blood vessels. The tonsillar crypts provided a complex edge against which alignment was possible, and the epithelial basement membrane followed its contour. We stained the blood vessels with an antibody to laminin that also stained the epithelial basement membrane. Therefore, by using the epithelial basement membrane-a normal constituent of the tissue-as the 
visually salient registration feature in the input CLSM image, we were able to align the tissue sections. Thus, CLSM images of tonsil tissue sections were used for $3 \mathrm{D}$ volume reconstruction accuracy evaluations.

\section{REGISTRATION METHODS}

As we described in the introduction, there are four registration steps. While certain parameters are defined once during registration of a batch of images, such as a reference coordinate system and a registration transformation model, other parameters have to be determined for each image separately, for example, locations of salient features and their spatial correspondences. Thus, our goal is to determine the most cost-efficient registration technique in terms of automation/labor and accuracy/time in order to automate selection of image-specific parameters. This leads us to the development of manual, semiautomatic, and fully automatic registration techniques based on algorithmic assumptions valid for a class of specimens imaged by CLSM.

There exist image mosaicking and alignment constraints that have been included in the software development as well. The current software has been developed for mosaicking problem constrained to spatial translations of image tiles and for image alignment problem constrained to affine transformation between two adjacent cross-sections. The description of the methods developed and evaluated in this work follows next.

\subsection{Image mosaicking}

Image mosaicking can be performed by visually inspecting spatially adjacent images, selecting one pair of corresponding points in the overlapping image area and computing transformation parameters for stitching together image tiles. This approach is denoted as manual mosaicking and is supported with software that enables (a) pixel selection of matching pairs of points and (b) computation of transformation parameters from a set of control points. If images are stitched together without any human intervention, then we refer to the method as automatic mosaicking. If a computer precomputes salient feature candidates and a user interaction specifies correspondences between any two features, then the method is referred to as semiautomatic mosaicking. Based on the underlying registration mechanism, we also denote manual registration as the pixel-based method and semiautomatic registration as the feature-based method.

First, we developed a manual mosaicking method that displays two spatially overlapping image tiles to a user. A user selects a pair of matching pixels, and then image tiles are stitched. In the next step, a user is presented with the already stitched image and a new tile to select matching pixels. Manual mosaicking is performed in this way till all images are stitched together and the final mosaicked image can be viewed for verification purposes. Second, we have developed a semiautomatic method that (1) highlights segmented vascular regions (closed contours) as salient feature candidates and (2) computes a pair of region centroids, as control points
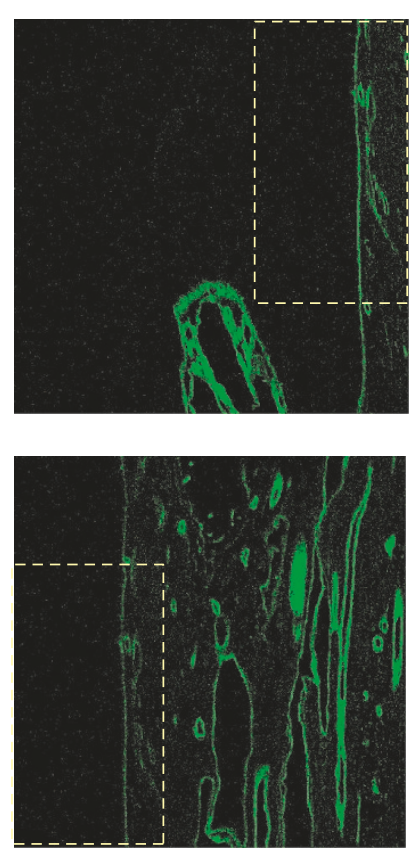

Figure 2: Adjacent tiles of CLSM images: overlapping regions have few vascular features.

for registration, after a user defined two region correspondences. This semiautomatic method is designed based on our observations that (a) an accurate point selection is much harder for a human than an accurate region (segment) selection, (b) a centroid selection of any region is less accurate by a human than by a computer, and (c) registration based on structural shape of a region rather than on intensity-defined point is more robust to noise. Third, we present a fully automatic mosaicking method. Full automation can be achieved by either automating feature-based registration process [1214] or maximizing pixel intensity correlation using computationally feasible search techniques with normalized crosscorrelation or mutual information metrics $[15,16]$.

To compare the mosaic accuracy, it would be more natural to achieve full automation by automating feature matching process. However, in CLSM imaging, it is not always feasible and the intensity-based methods have to be used. For example, there can be lack of detected vascular features in the overlapping region as it is illustrated in Figure 2. Although one might argue that intensity-based and feature-based techniques have different nature/principles behind their registration strategy, note that our objective is to evaluate registration accuracy which is independent of the chosen mosaicking technique. In our work, performing accuracy evaluations by comparing multiple techniques is independent of their underlying principles, and we focus only on their resulting mosaicking accuracy. One could also inspect Table 1 to realize that the intensity-based method (fully automatic) can lead to better or worse results than the feature-based method (manual and semiautomatic), which will be further discussed in Section 5.1. In this work, we demonstrate that the region centroid-based registration method significantly improves 
TABLE 1: A summary of mosaicking experiments: 3 by 3 tiles with 512 by 512 pixel resolutions. Full automatic methods are performed by the normalized cross-correlation (NC) and by the normalized mutual information (NMI) on pentium 4, 3.0 GHz.

\begin{tabular}{|c|c|c|c|c|c|c|}
\hline & \multicolumn{6}{|c|}{ Error (pixels) } \\
\hline & \multirow{2}{*}{\multicolumn{2}{|c|}{$\begin{array}{l}\text { Pixel-based } \\
\text { expert novice }\end{array}$}} & \multirow{2}{*}{\multicolumn{2}{|c|}{$\begin{array}{l}\text { Feature-based } \\
\text { expert novice }\end{array}$}} & \multicolumn{2}{|c|}{ Auto } \\
\hline & & & & & $\mathrm{NC}$ & NMI \\
\hline Average & 5.72 & 10.65 & 4.04 & 4.22 & 4.12 & 4.12 \\
\hline Standard deviation & 3.42 & 11.83 & 0.32 & 0.47 & & 0 \\
\hline Total average & \multicolumn{2}{|c|}{6.96} & \multicolumn{2}{|c|}{4.07} & \multicolumn{2}{|c|}{4.12} \\
\hline Total standard deviation & \multicolumn{2}{|c|}{6.82} & \multicolumn{2}{|c|}{0.35} & \multicolumn{2}{|l|}{0} \\
\hline \multirow[t]{4}{*}{ Upper bound (99.73\%) } & \multicolumn{2}{|c|}{27.42} & \multicolumn{2}{|c|}{5.12} & \multicolumn{2}{|c|}{4.12} \\
\hline & \multicolumn{6}{|c|}{ Time (seconds) } \\
\hline & \multirow{2}{*}{\multicolumn{2}{|c|}{$\begin{array}{l}\text { Pixel-based } \\
\text { expert novice }\end{array}$}} & \multirow{2}{*}{\multicolumn{2}{|c|}{$\begin{array}{l}\text { Feature-based } \\
\text { expert novice }\end{array}$}} & \multicolumn{2}{|c|}{ Auto } \\
\hline & & & & & $\mathrm{NC}$ & NMI \\
\hline Average & 211.56 & 153.47 & 125.27 & 101 & 68 & 480 \\
\hline Standard deviation & 132.32 & 95.06 & 56.96 & 45.66 & 0 & 0 \\
\hline Total average & & 7.03 & 11 & 9.2 & & 74 \\
\hline Total standard deviation & & 5.88 & & 5.01 & & 0 \\
\hline Upper bound (99.73\%) & & 1.67 & 284 & .23 & 27 & 74 \\
\hline
\end{tabular}

performance for 3D volume reconstruction of CLSM images in terms of achieved registration accuracy, consistency of the results, and performance time.

In our work, we used normalized mutual information and normalized cross-correlation metrics to find the best match of two tiles and to provide the sought translational offset for tile stitching. The main mosaicking advantages of these intensity correlation-based methods are (a) their relatively low computational cost for translation only, (b) robust performance for image tiles acquired with the same instrumentation setup, and (c) no user interaction (full automation). For example, Figure 3 shows how a high-resolution mosaicked image is constructed from nine image tiles.

\subsection{Image alignment}

Two challenges of image alignment include the transformation technique and model selection problems. In the past, the transformation technique based on correlation has been applied to many medical image modalities [17] other than the fluorescent CLSM modality. Nonetheless, applying the same techniques to the image alignment problem of CLSM images is more difficult due to (1) computational cost, (2) spatial intensity heterogeneity, and (3) noise issues as explained below.

First, the computational difficulty arises from a large image size and many degrees of freedom for complex transformation models. In our case, the computational complexity due to a large amount of data (3D stacks with many physical sections to obtain sufficient depth information) with high spatial resolution (around 2500 by 2500) should be considered when applying an affine transformation (6 degree of freedom). One could find methods in the literature that process large data of other imaging modalities than CLSM by using multiresolution- (or pyramid-) based techniques [17].

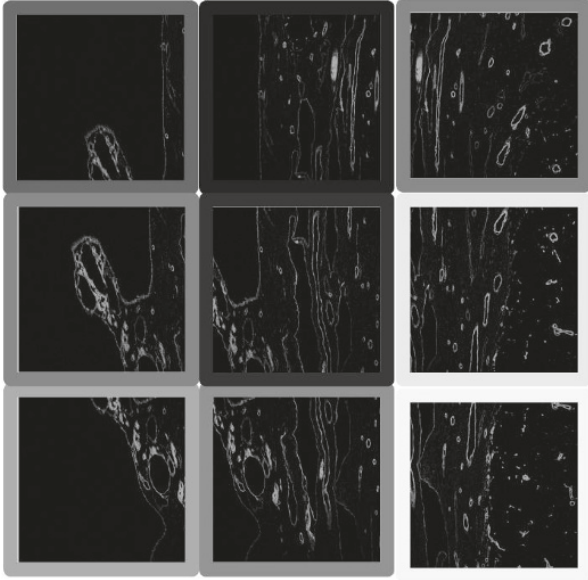

(a)

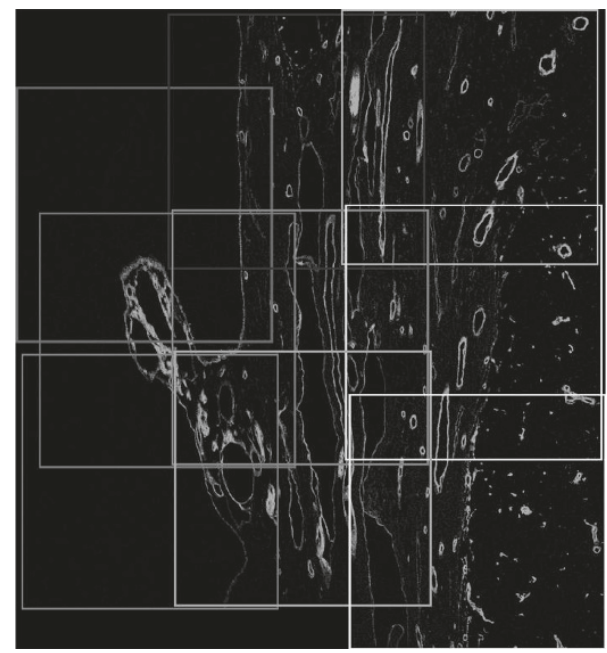

(b)

FIGURE 3: Image mosaicking problem: (a) image tiles with greyscale shaded borders and (b) mosaicked image showing where each tile belongs in the final image based on its grey-scale shade.

However, in the case of CLSM images, the local minima problem is more severe due to high spatial and depth intensity heterogeneity (attenuation) [18]. Second, varying signalto-noise ratio due to the aforementioned intensity heterogeneity should be considered. Third, noisy (spurious) features with high intensity values due to unbound fluorescence have to be handled.

To support our claims about spatial intensity heterogeneity and the presence of noise, we evaluated an intensity-based similarity metric (normalized correlation) for pairs of images from two registered CLSM subvolumes. The registration was conducted by semiautomated, region centroid-based alignment. The low magnitudes of these similarity values (approximately in the interval $[0.325,0.365]$ ) proved that the intensity-based automatic alignment would not be robust and would very frequently fail. As a consequence, we did not apply the correlation-based technique developed for image mosaicking to the image alignment problem. 
Furthermore, the problem of image alignment (or registration along $z$-axis) is much harder to automate than the problem of mosaicking because images of cross-sections are less similar than images of spatial tiles due to the process of cross-section specimen preparation (sample warping due to slicing), intensity variation (confocal imaging), and structural changes (bifurcating structures). In Figure 4, we quantified the morphological changes along depth in a single physical cross-section by computing normalized cross-correlation coefficient between the first and other image frames.

In terms of transformation model selection, higher-order (elastic), local or global models would be preferable to achieve smooth transition of image structures across slides (higher-order continuity). However, the difficulty with higher-order models is (a) in their robust parameter estimation due to intensity variation (noise) and deformation exceeding the order of the chosen model or (b) in bifurcation (appearing and disappearing structures). Although nonrigid optimization can be applied for only local features after a global alignment, we limited our transformation model as an affine because the transformations using higher-order models could lead to erroneous alignment due to the well-known leaning tower problem [19], and could ultimately distort the $3 \mathrm{D}$ anatomical structures (features) by matching accurately small regions while significantly distorting other regions.

Rigid transformation model with only translation and rotation is one of the most popular lower-order transformation models designed for rigid structures like bones. However, in our case, the paraffin-embedded tonsil tissue represents a nonrigid structure and has to include deformation like shear due to tissue slicing. Considering the medical specimens of our interest, we chose an affine transformation for modeling cross-section distortions and expected to detect only a small amount of scale and shear deformations. We plan to research automatic registration techniques using other transformation models in future.

Given the affine transformation model $\alpha: \mathbb{R}^{2} \rightarrow \mathbb{R}^{2}$, the image alignment can be performed by selecting at least three pairs of corresponding points and computing six affine transformation parameters shown below:

$$
\left[\begin{array}{l}
x^{\prime} \\
y^{\prime}
\end{array}\right]=\left[\begin{array}{ll}
a_{00} & a_{01} \\
a_{10} & a_{11}
\end{array}\right]\left[\begin{array}{l}
x \\
y
\end{array}\right]+\left[\begin{array}{l}
t_{x} \\
t_{y}
\end{array}\right]
$$

The $\left(x^{\prime}, y^{\prime}\right)=\alpha(x, y)$ values are the transformed coordinates $(x, y)$. The four parameters, $a_{00}, a_{10}, a_{01}$, and $a_{11}$, represent a 2 by 2 matrix compensating for scale, rotation, and shear distortions in the final image. The two parameters, $t_{x}$ and $t_{y}$, represent a $2 \mathrm{D}$ vector of translation.

The manual and semiautomatic methods for image alignment differ from the methods described for image mosaicking by the need to select at least three pairs of corresponding registration points as opposed to one pair of points sufficient in the case of image mosaicking. The affine transformation parameters are computed by solving six or more linear equations.

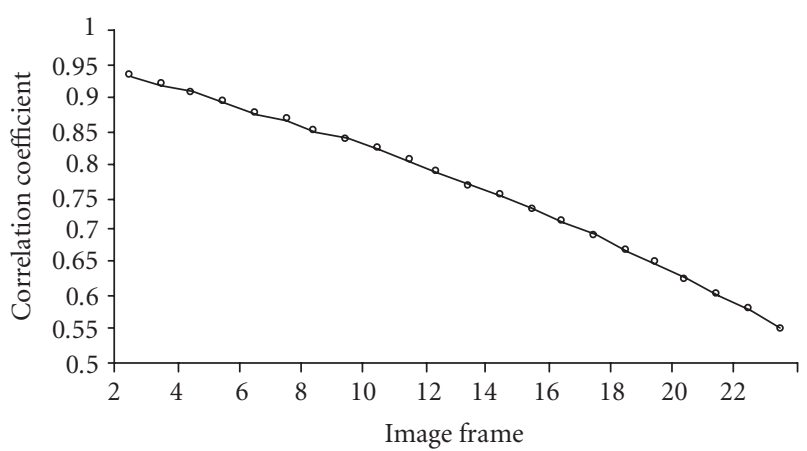

FIGURE 4: Morphology quantification in a CLSM stack: $x$-axis represents a frame index (along depth) which is compared with the first frame.

\section{EVALUATION METHODOLOGY FOR REGISTRATION ACCURACY}

In this section, we outline our methodology for assessing upper error bounds of automatic, semiautomatic, and manual 3D volume reconstruction techniques. Our experimental variables include (1) the type of registration problem (image mosaicking and alignment), (2) the type of registration method (automatic, semiautomatic, and manual), and (3) the type of human subject (experts and novices) doing registration. Human subjects were labeled as experts if they had the knowledge about CLSM imaging, imaged specimen and its anatomical/structural properties, and/or principles of the affine transformation-based registration algorithm. This type of knowledge was critical for establishing feature correspondences and obtaining accurate registration results.

Our primary evaluation criterion is registration accuracy with an auxiliary measure of performance time. The challenges of registration evaluations are usually in defining optimality criteria for assessing registration accuracy and in knowing the ground truth (or a reference image). The two fundamental questions that arise during registration accuracy evaluations are (1) what to compare the registered (mosaicked or aligned) image to, and (2) how to compare two images. Next, we describe how these challenges were overcome for image mosaicking and image alignment accuracy evaluations.

\subsection{Image mosaicking accuracy evaluation}

In the case of image mosaicking, we could carve out several spatially overlapping tiles from one large image and use the original image as the reference (ground truth) image. However, this evaluation setup would not simulate the real problem of mosaicking multiple tiles acquired at different time instances, and therefore would not represent unpredictable intensity variations due to fluorescent imaging physics. Thus, we chose to establish the ground truth image and the locations of all $n$ tiles in this image (denoted as $T^{\mathrm{GT}}$ in (2)) in the following way. 
First, we took an overview image of a specimen at $20 \times$ optical magnification and $3 \times 3$ high-resolution image tiles at $63 \times$ optical magnification $(n=9)$. The overview image became the ground truth image. Second, tile images (63x magnification) are digitally downsampled to match the resolution of the overview image (20× magnification). Third, we find the best match between a downsampled tile and the overview image with a template-based search technique using a normalized cross-correlation metric. Fourth, the location of the best tile match is rescaled to the original tile resolution. Fifth, steps one through four are repeated for all nine tiles to obtain a matrix of tile locations $T^{*}$. Sixth, the matrix $T^{*}$ is normalized with respect to the tile location in the upper left corner $\left(t_{1 x}, t_{1 y}\right)$ of the final mosaic image. Note that we have used a bilinear interpolation method for down- and upsampling processes.

The uncertainty (pixel error distance) caused by the resampling (e.g., interpolation) procedure can be easily computed from the magnification factors. For example, for the resampling factor equal to $63 / 20(=3.15)$, a downsampled pixel will have contributions from a 3.15 by 3.15 pixel neighborhood. Thus, the uncertainty of the downsampled and rescaled pixel is equal to the maximum pixel distance in a 3.15 by 3.15 pixel region $\left(3.04(=2.15 \sqrt{2})\right.$ pixels $\left.^{1}\right)$.

We denote the normalized matrix as the ground truth matrix $T^{\mathrm{GT}}$ of tile locations:

$$
\begin{array}{r}
T^{\mathrm{GT}}=\left(\begin{array}{cc}
t_{1 x}^{\mathrm{GT}} & t_{1 y}^{\mathrm{GT}} \\
t_{2 x}^{\mathrm{GT}} & t_{2 y}^{\mathrm{GT}} \\
\vdots & \vdots \\
t_{n x}^{\mathrm{GT}} & t_{n y}^{\mathrm{GT}}
\end{array}\right)=T^{*}-\left(\begin{array}{cc}
t_{1 x} & t_{1 y} \\
t_{1 x} & t_{1 y} \\
\vdots & \vdots \\
t_{1 x} & t_{1 y}
\end{array}\right), \\
\text { where } T^{*}=\left(\begin{array}{cc}
t_{1 x} & t_{1 y} \\
t_{2 x} & t_{2 y} \\
\vdots & \vdots \\
t_{n x} & t_{n y}
\end{array}\right) .
\end{array}
$$

Any other result of mosaicking is represented by a matrix of tile locations $T$ and compared with $T^{\mathrm{GT}}$. The mosaicking registration error $E_{\text {translation }}$ is computed as an average error distance according to the formula in (3). Note that the smaller the error implies the better mosaicking accuracy:

$$
E_{\text {translation }}=\frac{1}{n} \sum_{i=1}^{n} \sqrt{\left(t_{i x}^{\mathrm{GT}}-t_{i x}\right)^{2}+\left(t_{i y}^{\mathrm{GT}}-t_{i y}\right)^{2}}
$$

The proposed mosaicking evaluation methodology using (1) the overview image acquired at low optical magnification as the true reference image and (2) the normalized correlation-based estimation of tile locations $T^{\mathrm{GT}}$ simulates more closely real image tile data than a set of carved out tiles from one image. Furthermore, the bias of tile locations

\footnotetext{
${ }^{1}$ Note. Geometrically, the maximum distance is a Euclidean distance between the centers of pixels in a region.
}

$T^{\mathrm{GT}}$ coming from normalized correlation-based matching can be quantitatively expressed by the correlation values in the vicinity of the best tile match with the overview image. Our final remark is related to the selection of the error metric $E_{\text {translation. }}$. Due to the intensity variations of CLSM images, it is preferable to use a registration accuracy metric based on spatial matches of salient structures rather than on pixel intensity matches. The appropriateness of this metric selection could be demonstrated by taking images of the same specimen multiple times without moving it. If the metric would be based on pixel intensity matches, then the metric would indicate falsely misregistration in contrary to the metric based on spatial matches.

\subsection{Image alignment accuracy evaluation}

Similarly to the case of image mosaicking, we could create a pair of misaligned images by applying a known affine transformation to any image and presenting the original and transformed images to a user for accuracy evaluation purposes. However, this evaluation setup would not simulate the real problem of image alignment where two cross-sections might have missing or new or warped structures with a priori unknown intensity variations. Thus, we chose to establish the reference image and its corresponding affine transformation parameters in the following way.

First, we acquired a stack of CLSM images that are coregistered along $z$-axis because a specimen has not moved while the focal depth of CLSM has varied during image acquisition. Second, multiple stacks of CLSM images are aligned by a manual alignment method and the representative of all resulting affine transformations is recorded, for example, maximum translation, rotation, and shear. Third, a pair of misaligned images is constructed for accuracy evaluations by taking the first and last images along the $z$-axis of one CLSM physical section and applying the representative affine transformation (recorded in step 2) to the last image. The first and the last transformed images become the evaluation images with the known ground truth affine transformation $\alpha^{\mathrm{GT}}(\cdot)$. All pixel coordinates of the transformed (ground truth) image $\mathbf{P}^{\mathrm{GT}}=\left\{p_{1}^{\mathrm{gt}}, p_{2}^{\mathrm{gt}}, \ldots, p_{n}^{\mathrm{gt}}\right\}$ are then defined by the affine transformation $\alpha^{\mathrm{GT}}: p_{i} \rightarrow p_{i}^{\mathrm{gt}}$. Based on user's registration input, an affine transformation $\alpha^{\mathrm{USR}}(\cdot)$ is estimated. We denote the corresponding set of transformed pixel coordinates as $\mathbf{P}^{\mathrm{USR}}=\left\{p_{1}^{\mathrm{usr}}, p_{2}^{\mathrm{usr}}, \ldots, p_{n}^{\text {usr }}\right\}$, where $\alpha^{\mathrm{USR}}: p_{i} \rightarrow p_{i}^{\mathrm{usr}}$. The final image alignment registration error $E_{\text {affine }}$ is then calculated as an average Euclidean error distance over all pixels coordinates according to (4), where $m$ is the number of transformed pixels. Once again, with the smaller the error $E_{\text {affine, }}$, the better image alignment accuracy is achieved:

$$
E_{\text {affine }}=\frac{1}{m} \sum_{i=1}^{m} \sqrt{\left(p_{i x}^{\mathrm{gt}}-p_{i x}^{\mathrm{usr}}\right)^{2}+\left(p_{i y}^{\mathrm{gt}}-p_{i y}^{\mathrm{usr}}\right)^{2}} .
$$

The proposed image alignment evaluation methodology utilizes (1) confocal imaging to obtain required image frames, and (2) empirically observed affine distortions to prepare test alignment data as close to real data as possible. 
The justification for choosing the alignment error metric $E_{\text {affine }}$ is twofold. First, similar to the explanation provided for the choice of the mosaicking error metric, an error metric based on pixel locations seems more appropriate than a metric based on intensity comparisons due to CLSM intensity variations. Second, it would not be fair to compute differences of affine transformation parameters since they represent a mix of distortions (translation, rotation, scale, and shear). Euclidean distances over the registered area reflect the degree of misalignment. It would be possible to consider a metric that would include the spatial mismatch only over the set pixels that are above a certain intensity threshold. However, we decided to avoid introducing a threshold parameter into our evaluation metric due to different unknown intensity ranges and distributions of a pair of compared images.

\subsection{Statistical performance evaluation}

Now we describe a statistical test method to evaluate accuracy improvement of the feature-based approach against pixelbased approach. Let $\left\{E_{i}^{P}\right\}$ and $\left\{E_{i}^{F}\right\}$ be two paired sets of $N$ measured error values for the pixel-based method and the feature-based method, respectively, obtained with the same data. In our experiments, the size of the set is relatively large ( $N=50$ for mosaicking and $N=78$ for alignment). We assume that the paired error values are independent and follow a Gaussian distribution. The null hypothesis in our tests states that there is no improvement of the feature-based registration approach in comparison with the pixel-based registration approach. We perform the Student $t$ test to prove or reject the null hypothesis [20]. We compute $\hat{E}_{i}^{P}=\left(E_{i}^{P}-\bar{E}_{i}^{P}\right)$ and $\hat{E}_{i}^{F}=\left(E_{i}^{F}-\bar{E}_{i}^{F}\right)$, where $\bar{E}_{i}^{P}$ and $\bar{E}_{i}^{F}$ are the average errors of each set. Then, we calculate the $t$ value for the paired $t$ test according to the equation below:

$$
t=\left(\bar{E}^{P}-\bar{E}^{F}\right) \sqrt{\frac{N(N-1)}{\sum_{i=1}^{N}\left(\hat{E}_{i}^{P}-\hat{E}_{i}^{F}\right)^{2}}} .
$$

Given the $t$ value from (5), we obtain the confidence interval ( $p$ value [20]) to prove or reject the null hypothesis (no improvement) using one-tailed cumulative probability distribution function $P(X \leq t)$ with $N-1$ degrees of freedom. The results of statistical comparisons are shown in the next section.

\section{EXPERIMENTAL RESULTS}

The overall experiments consisted of mosaicking $3 \times 3$ image tiles (see Figure 3 ) and aligning three pairs of different crosssections (see image examples in Figure 5). We report results obtained from twenty human subjects (fifteen experts and five novices) who participated in our study, and performed manual and semiautomatic image mosaicking and alignment registrations. To assess registration consistency, novices performed registration three times with any given data set. Although the results from novices may be biased by "a learning effect," we did not observe it in our experiments due to the small number of trial repetitions.
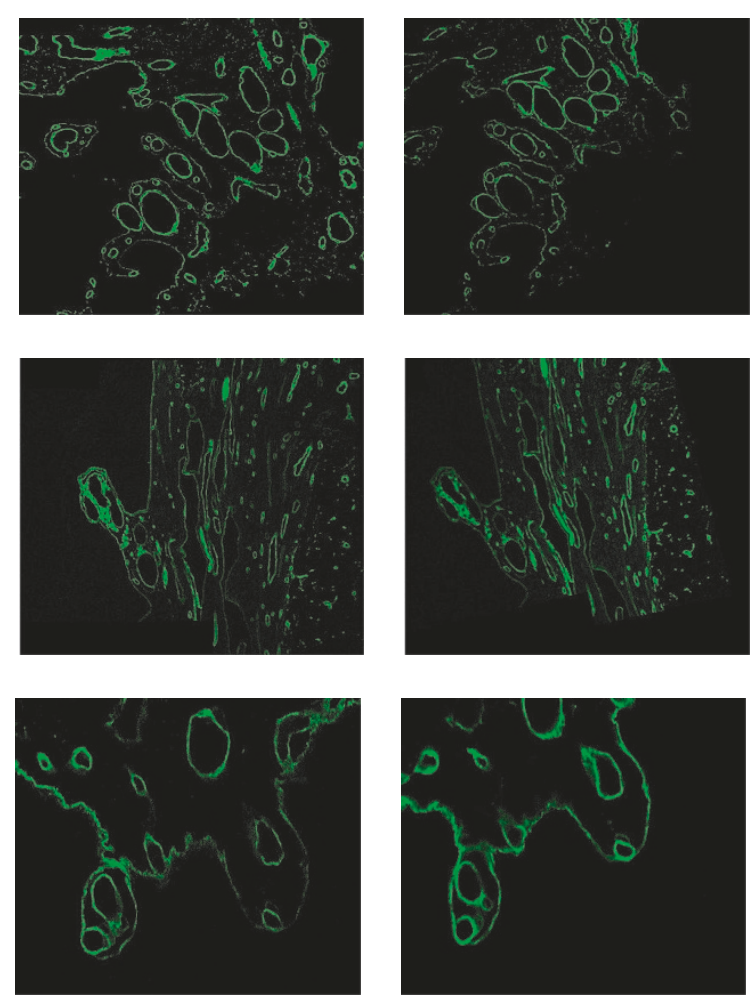

FIgure 5: Three pairs (top), (middle), and (bottom) of image examples used for alignment evaluation. (Left) Reference image from the first frame. (Right) Transformed image of the last frame based on predefined affine transformation.

\subsection{Image mosaicking}

Figure 6(a) shows the user interface for selecting matching points in two image tiles. Users selected one pair of feature points, one from each tile. Figure 6(b) illustrates the interface for selecting regions that would be used for centroid calculation. In order to construct a mosaicked image (as shown in Figure 3), eight pairs of points or regions had to be selected. We used a set of nine images from a single physical section for mosaicking, and the experimental results are summarized in Figure 7 and Table 1, and the $t$ test result comparing the pixel-based and feature-based mosaicking is shown in Table 2.

Tables 1 and 2 lead to the following conclusions. First, fully automatic mosaicking using normalized cross-correlation similarity is the fastest method, followed by semiautomatic (feature-based) and manual mosaicking. Second, manual pixel-based image mosaicking is the least accurate with the highest standard deviation among all methods. Third, semiautomatic and fully automatic mosaicking methods are approximately equally accurate. Fourth, experts using the manual (pixel-based) mosaicking method selected one pair of points/regions more accurately (small average error) and consistently (small standard deviation) than novices although it took them more time. Fifth, the difference in mosaicking average errors and their standard deviations between experts and novices using the pixel-based method 


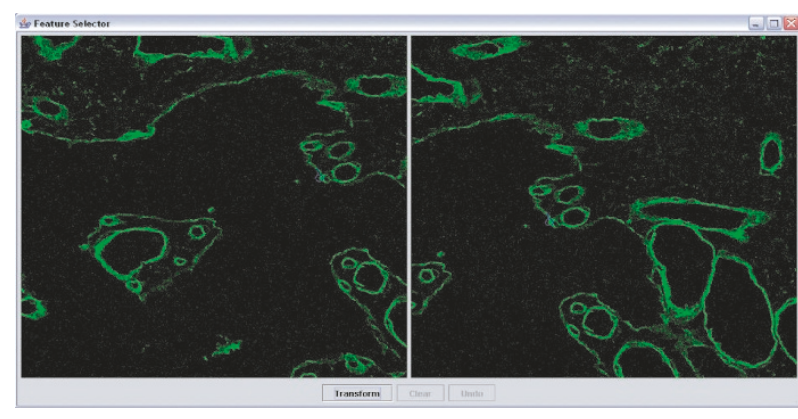

(a)

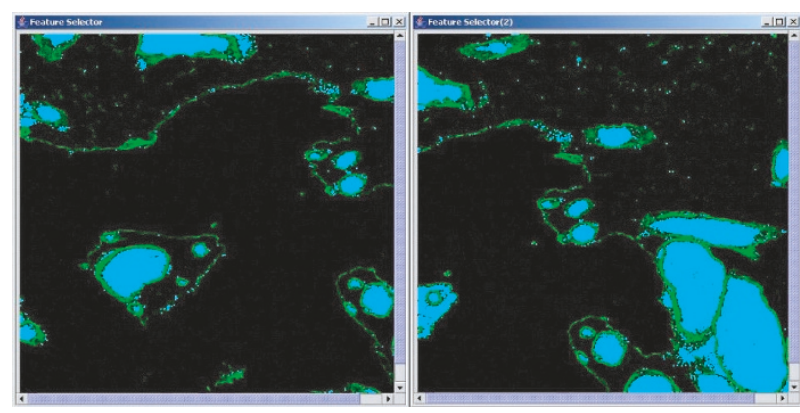

(b)

FIgURE 6: Software interface for (a) manual mosaicking and (b) semiautomatic mosaicking with highlighted regions.

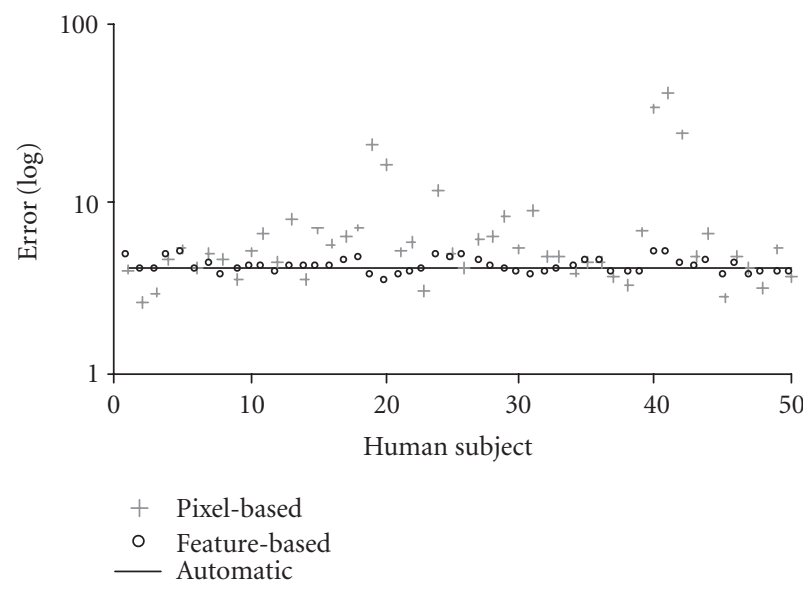

FIGURE 7: Mosaicking registration errors for all human subjects performing pixel-based (manual) and feature-based (semiautomatic) tile mosaicking computed according to (3).

disappears when human subjects start using the featurebased mosaicking method. Sixth, the upper error bound of each mosaicking method can be estimated in pixels as the average plus three times standard deviation (99.73\% confidence interval), which leads to about $4.12,5.12$, and 27.42 pixel errors for the fully automatic, semiautomatic, and manual methods, respectively. Seventh, the $t$ test result in Table 2 shows that the null hypothesis (no improvement) is rejected with $99.8 \%$ confidence. Finally, the timesaving for experts
TABLE 2: The paired $t$ test result for errors of the pixel-based and the feature-based methods in Table 1.

\begin{tabular}{lc}
\hline \multicolumn{2}{c}{ Pixel-based versus feature-based } \\
\hline Degrees of freedom & 49 \\
$t$ value & 3.019 \\
$p$ value & 0.998 \\
\hline
\end{tabular}

and novices using semiautomatic method with respect to manual method is $41 \%$ and $36 \%$, respectively.

Although the feature-based semiautomatic methods or the intensity-based automatic methods look pretty attractive, note that there are mosaicking cases when the overlapping area of two adjacent tiles is characterized by either a lack of detected vascular features (feature-based techniques fail) or significant spatial intensity heterogeneity (intensitybased techniques fail). Figure 2 illustrates the former case. Thus, there is a need to evaluate manual and semi-automated mosaicking techniques for those cases when the intensitybased techniques fail. In addition, it is not always the case that the fully automatic method will outperform the manual and semiautomatic methods (see Table 1).

\subsection{Image alignment}

For the image alignment experiments, we used the same user interfaces for selecting multiple points and regions as shown in Figure 6. We recommended that human subjects select at least three points or regions, in such a way that they would be well spatially distributed in each image but would not be collinear. If points are close to be collinear, then the affine transformation parameters cannot be uniquely derived from a set of linear equations (more unknowns than the number of equations), which leads to large alignment errors. If points are locally clustered and do not cover an entire image spatially, then the affine transformation is very accurate only in the proximity of the selected points. However, the affine transformation inaccuracy increases with the distance from the selected points, which leads to large alignment error since the error metric takes into account errors across the entire image area. In order to assess the points selected by a user in terms of their distribution and collinear arrangement, we have designed a compactness measure defined as a ratio of the entire image area $A_{\text {Image }}$ divided by the largest triangular area $a_{\text {Triangle }}$ formed from three points in the selected points (see (6)):

$$
\text { Compactness Measure }=A_{\text {Image }} / a_{\text {Triangle }} .
$$

We observed large alignment error when human subjects selected almost collinear points or locally clustered points regardless of our recommendations. Figure 8 shows the relationship between compactness and alignment error modeled with a linear fit. We used three different pairs of adjacent physical sections for alignment study, and the error results of all experiments as a function of human subject trials are shown in Figure 9 and summarized in Table 3. The $t$ test values for comparing the pixel-based and feature-based mosaicking are shown in Table 4. 


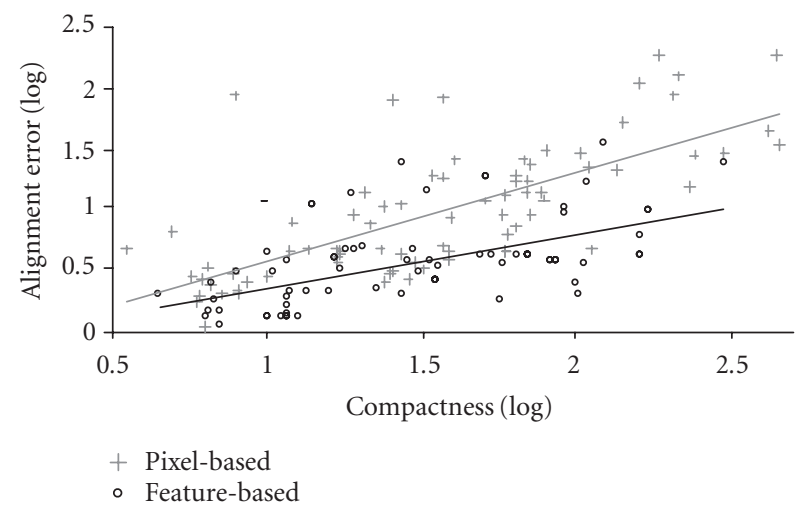

Figure 8: Illustration of a strong correlation between the compactness measure and the alignment error.

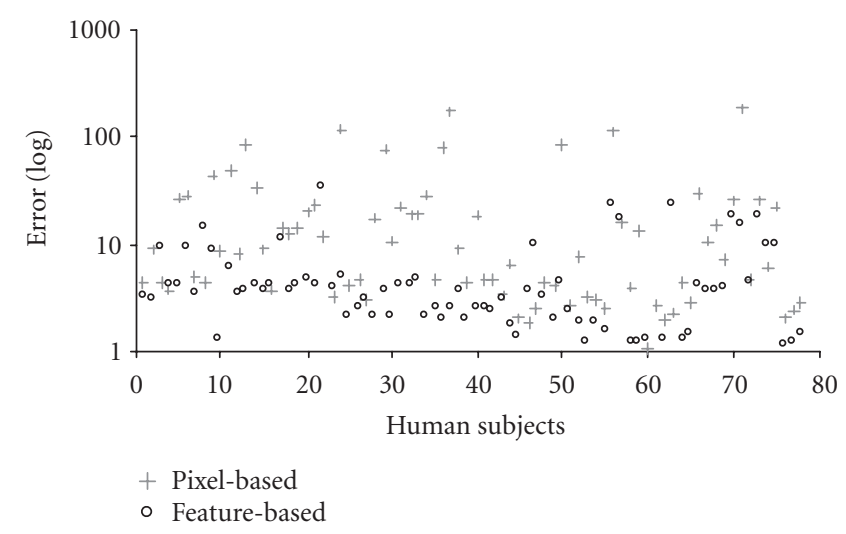

FIgURE 9: Alignment errors for all human trials including pixelbased (manual) and feature-based (semiautomatic) alignment.

The image alignment results in Figure 8 and Table 3 lead us to the following conclusions. First, manual (pixel-based) image alignment is less accurate and less consistent (large standard deviation) than the semiautomatic (feature-based) alignment. Based on the $t$ test result in Table 4 , the null hypothesis (no improvement) can be rejected with $99.9 \%$ confidence. Second, selection of (a) collinear features or (b) spatially dense points or regions can have a detrimental effect on alignment accuracy. Third, experts achieved higher average alignment accuracy than novices with both methods. Finally, the difference in alignment errors between experts and novices using the pixel-based method is significantly reduced when human subjects start using the feature-based alignment method. We should also mention that the majority of human subjects selected only three points or regions for aligning two images. To demonstrate the effect of the number of selected points on the registration accuracy, we computed the accuracy by using all matching pairs of features detected by segmentation $(27,21$, and 4 pairs for each test in Figure 5). The estimated affine transformation results in $1.21,1.12$, and 2.54 pixel error distances for each test data, respectively. The average pixel error distance is equal to 1.62 pixels and the standard deviation is 0.79 . This result indicates
TABLE 3: A summary of image alignment.

\begin{tabular}{|c|c|c|c|c|}
\hline Error (pixels) & $\begin{array}{r}\text { Pixel } \\
\text { expert }\end{array}$ & $\begin{array}{l}\text {-based } \\
\text { novice }\end{array}$ & $\begin{array}{l}\text { Featur } \\
\text { expert }\end{array}$ & $\begin{array}{r}\text { e-based } \\
\text { novice }\end{array}$ \\
\hline Average & 17.32 & 27.98 & 4.85 & 5.83 \\
\hline Standard deviation & 27.12 & 43.28 & 5.63 & 6.71 \\
\hline Total average & \multicolumn{2}{|c|}{22.28} & \multicolumn{2}{|c|}{5.28} \\
\hline Total standard deviation & \multicolumn{2}{|c|}{35.74} & \multicolumn{2}{|c|}{6.11} \\
\hline Upper bound ( $99.73 \%$ confidence) & \multicolumn{2}{|c|}{129.5} & \multicolumn{2}{|c|}{23.61} \\
\hline
\end{tabular}

TABLE 4: The paired $t$ test result for errors of the pixel-based and the feature-based methods in Table 3.

\begin{tabular}{ll}
\hline \multicolumn{2}{c}{ Pixel-based versus feature-based } \\
\hline Degrees of freedom & 77 \\
$t$ value & 4.109 \\
$p$ value & 0.999 \\
\hline
\end{tabular}

that (a) more well-matched points lead to more accurate alignment, and (b) instructing human subjects to choose the maximum number of the features detected by segmentation would lead to higher alignment accuracy.

\subsection{Discussion of mosaicking and alignment results}

We investigate the main factors behind the summarized experimental results and present them in this section. First, the feature-based registration is faster and more accurate than pixel-based registration for both mosaicking and alignment problems. Our confidence in accuracy improvement is supported by the paired $t$ test result. We did not report time measurements for the alignment problem because the experiments were conducted on multiple computers with different operating speeds and the reported numbers for mosaicking provide only indications of true comparative values.

Second, the image alignment upper bound errors (23.61 for semiauto and 129.5 for manual) are much higher than the mosaicking upper bound errors (4.12 for auto, 5.12 for semiauto, and 27.42 for manual). We believe that the main factors behind these differences are (1) a higher-order complexity of the alignment problem (intensity and spatial structure variations across slides) in comparison with the mosaicking problem (intensity variations across tiles), (2) a larger degree of freedom in occurring image alignment transformations (rotation, scale, shear, and translation) than in mosaicking transformations (translation), and (3) significantly larger sensitivity to human inconsistency in selecting points (attention level, skills, fatigue, display quality). Human inconsistency is expressed by a much larger standard deviation of errors in the case of alignment (35.74 for manual and 6.11 for semiauto) than in the case of mosaicking ( 6.82 for manual and 0.35 for semiautomatic).

In addition, we would like to add a few comments about the performance robustness of fully automatic and semiautomatic methods. Fully automatic mosaicking method based 
on normalized correlation or normalized mutual information might not achieve the best performance when corresponding salient features have spatially mismatched intensity variations. Semiautomatic method based on region centroids might not be used when closed regions cannot be detected due to the spatial structure of an imaged specimen or a very low image quality, for instance, a small signal-to-noise (SNR) ratio and a large amount of intraregion noise. We will investigate in future how to predict accurately centroids of partially open regions and closed regions with speckle noise internal to a region.

\section{CONCLUSIONS}

We presented an accuracy evaluation of 3D volume reconstruction from CLSM imagery that consists of image mosaicking and image alignment registration steps. The contribution of this paper is not only in developing three registration methods having different levels of automation but also in proposing a methodology for conducting realistic evaluations and performing a thorough analysis of the experimental results. We report accuracy evaluations for (1) three registration methods including manual (pixel-based), semiautomatic (region centroid feature-based), and fully automatic (correlation-based) registration techniques, (2) two groups of human subjects (experts and novices), and (3) two types of registration problems (mosaicking and alignment). Our study demonstrates significant benefits of automation for $3 \mathrm{D}$ volume reconstruction in terms of achieved accuracy, consistency of results, and performance time. In addition, the results indicate that the differences between registration accuracy obtained by experts and by novices disappear with an advanced automation while the absolute registration accuracy increases. If one is interested in performing data-specific evaluations, then we prepared webbased tools [21] for better data understanding and analysis at http://isda.ncsa.uiuc.edu/MedVolume/.

\section{ACKNOWLEDGMENTS}

This material is based upon work supported by the National Institute of Health under Grant no. R01 EY10457. The ongoing research is a collaboration between the Department of Pathology, College of Medicine, University of Illinois at Chicago, and the Automatic Learning Group, National Center for Supercomputing Applications, University of Illinois at Urbana-Champaign.

\section{REFERENCES}

[1] S.-C. Lee and P. Bajcsy, "Feature based registration of fluorescent LSCM imagery using region centroids," in Medical Imaging, vol. 5747 of Proceedings of the SPIE, pp. 170-181, San Diego, Calif, USA, February 2005.

[2] C. L. Collins, J. H. Ideker, and K. E. Kurtis, "Laser scanning confocal microscopy for in situ monitoring of alkali-silica reaction," Journal of Microscopy, vol. 213, no. 2, pp. 149-157, 2004.
[3] J. B. Pawley, The Handbook of Biological Confocal Microscopy, Plenum Press, New York, NY, USA, 1990.

[4] B. Zitova and J. Flusser, "Image registration methods: a survey," Image and Vision Computing, vol. 21, no. 11, pp. 9771000, 2003.

[5] D. L. G. Hill, P. G. Batchelor, M. Holden, and D. J. Hawkes, "Medical image registration," Physics in Medicine and Biology, vol. 46, no. 3, pp. R1-R45, 2001.

[6] L. G. Brown, "A survey of image registration techniques," ACM Computing Surveys, vol. 24, no. 4, pp. 325-376, 1992.

[7] L. D. Cohen and I. Cohen, "Deformable models for 3-D medical images using finite elements and balloons," in Proceedings of IEEE Computer Society Conference on Computer Vision and Pattern Recognition (CVPR '92), pp. 592-598, Champaign, Ill, USA, June 1992.

[8] A. Goshtasby, "Registration of images with geometric distortions," IEEE Transactions on Geoscience and Remote Sensing, vol. 26, no. 1, pp. 60-64, 1988.

[9] M. Tuohy, C. A. McConchie, R. B. Knox, L. Szarski, and A. Arkin, "Computer-assisted three-dimensional reconstruction technology in plant cell image analysis; applications of interactive computer graphics," Journal of Microscopy, vol. 147, no. 1, pp. 83-88, 1987.

[10] J. B. A. Maintz and M. A. Viergever, "A survey of medical image registration," Medical Image Analysis, vol. 2, no. 1, pp. 1-36, 1998.

[11] A. Nocito, J. Kononen, O. P. Kallioniemi, and G. Sauter, "Tissue microarrays (TMAs) for high-throughput molecular pathology research," International Journal of Cancer, vol. 94, no. 1, pp. 1-5, 2001.

[12] A. Goshtasby, G. C. Stockman, and C. V. Page, "A region-based approach to digital image transformation with subpixel accuracy," IEEE Transactions on Geoscience and Remote Sensing, vol. 24, no. 3, pp. 390-399, 1986.

[13] J. Flusser and T. Suk, "A moment-based approach to registration of images with affine geometric distortion," IEEE Transactions on Geoscience and Remote Sensing, vol. 32, no. 2, pp. 382-387, 1994.

[14] H. Li, B. S. Manjunath, and S. K. Mitra, "A contour-based approach to multisensor image registration," IEEE Transactions on Image Processing, vol. 4, no. 3, pp. 320-334, 1995.

[15] W. K. Pratt, "Correlation techniques of image registration," IEEE Transactions on Aerospace and Electronic Systems, vol. 10, no. 3, pp. 353-358, 1974.

[16] P. Viola and W. M. Wells III, "Alignment by maximization of mutual information," in Proceedings of the 5th International Conference on Computer Vision (ICCV'95), pp. 16-23, Cambridge, Mass, USA, June 1995.

[17] G. Hermosillo, C. Chefd'Hotel, and O. Faugeras, "Variational methods for multimodal image matching," International Journal of Computer Vision, vol. 50, no. 3, pp. 329-343, 2002.

[18] M. Jungke, W. Von Seelenon, G. Bielke, et al., "A system for the diagnostic use of tissue characterizing parameters in NMRtomography," in Proceedings of Information Processing in Medical Imaging (IPMI'87), vol. 39, pp. 471-481, 1987.

[19] K. Montgomery and M. D. Ross, "Non fiducial, shaped-based registration of biological tissue," in Three-Dimensional Microscopy: Image Acquisition and Processing III, vol. 2655 of Proceedings of SPIE, pp. 224-232, San Jose, Calif, USA, January 1996.

[20] C. H. Goulden, Methods of Statistical Analysis, John Wiley \& Sons, New York, NY, USA, 2nd edition, 1956. 
[21] R. Kooper, A. Shirk, S.-C. Lee, A. Lin, R. Folberg, and P. Bajcsy, "3D medical volume reconstruction using Web services," in Proceedings of IEEE International Conference on Web Services (ICWS '05), p. 716, Orlando, Fla, USA, July 2005.

Sang-Chul Lee received his M.S. degree in computer science from the University of Illinois at Urbana-Champaign in 2003 and his B.E. degree in computer science and engineering from the Inha University, Korea, in 1998. His research interests are in computer vision, robotics, and medical imaging. His research projects include omnidirectional vision-based mobile robot navigation, data fusion for NEES (Network for

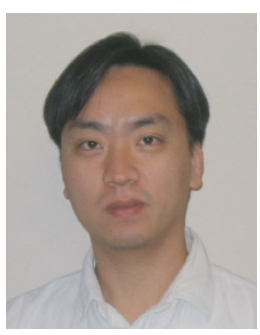
Earthquake Engineering Simulation), and 3D volume reconstruction of CLSM imagery (in collaboration with the Department of Pathology, University of Illinois at Chicago). He is currently a Research Assistant in the Automated Learning Group, National Center for Supercomputing Applications (NCSA), and a Ph.D. candidate in computer science at University of Illinois at UrbanaChampaign.

Peter Bajcsy has earned his Ph.D. degree from the Electrical and Computer Engineering Department, University of Illinois at Urbana-Champaign, Ill, 1997, and M.S. degree from the Electrical Engineering Department, University of Pennsylvania, Philadelphia, Pa, 1994. He is currently with the National Center for Supercomputing Applications at the University of Illinois at Urbana-Champaign, Illinois, working as

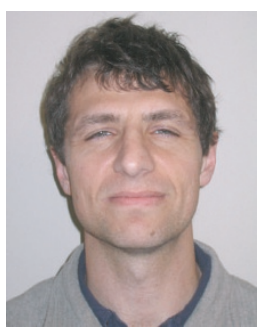
a Research Scientist on problems related to automatic transfer of image content to knowledge. In the past, he had worked on realtime machine vision problems for semiconductor industry and synthetic aperture radar (SAR) technology for government contracting industry. He has developed several software systems for automatic feature extraction, feature selection, segmentation, classification, tracking, and statistical modeling from electrooptical, SAR, laser, and hyperspectral data sets. His scientific interests include image and signal processing, statistical data analysis, data mining, pattern recognition, novel sensor technology, and computer and machine vision.

Amy Lin received her undergraduate degree in biomedical engineering and M.D. degree from Johns Hopkins University. She is a Board Certified Ophthalmologist, having completed residency training in this discipline from the University of Illinois Department of Ophthalmology and Visual Sciences. While collaborating on this research project, she was the Knapp Fellow in ophthalmic pathology at the University of Illinois at Chicago (UIC). She is currently serving as a Resident Physician in pathology at UIC.

Robert Folberg received his undergraduate degree from LaSalle University and his M.D. degree from Temple University. He holds Board certification in ophthalmology and anatomic pathology. He is currently the Frances B. Geever Professor and Head of the Department of Pathology at the University of Illinois at Chicago. His research focuses in part on the the three-dimensional distribution of extracellular matrix proteins in uveal melanoma. 